MODELING, IDENTIFICATION AND CONTROL, 1982, vOL. 3, NO. 4, 223-230

doi:10.4173/mic.19824.3

\title{
Compounding the losses of convoyed ships attacked by tactical submarines
}

\author{
K. M. MJELDE†
}

Keywords: defence analysis, differential games, numerical modeling.

It is demonstrated that a time dependent stochastic model for the losses of convoyed ships attacked by submarines can be applied in the determination of the losses in terms of the results of two submodels:

(1) a model of the tactical options of an individual submarine captain and

(2) a new model of the total losses of ships inflicted by a single submarine in the time interval since arrival at the operating area.

This approach gives simplicity and structure to equations that would, otherwise, easily become complicated, except in special cases, such as a previously considered case of submarines carrying weapons of a single type. An example of a scenario with submarines releasing torpedoes and missiles is considered, and applied in the motivation of the definitions of the quantities of submodel 2 above. Various applicational aspects are commented on.

\section{Introduction}

A model for the time dependent probability distribution of the total losses of convoyed merchant ships attacked by submarines was given by Mjelde (1975), and applied to the case of each submarine releasing a maximal number of loads of a weapon of a single type (torpedoes or missiles). The analysis of more complex scenarios, such as submarines releasing missiles and torpedoes, frequently leads to equations that are algebraically complicated, unless suitably structured. It is the purpose of this paper to present such a structured set of equations for the time dependent total stochastic losses due to several submarines, in terms of two sub-models:

(1) a model describing the tactical options of an individual submarine captain, and

(2) a new model for the composition of the number of ships destroyed due to several attacks by an individual submarine.

The total number of ships destroyed by all submarines is expressed in terms of the results of the submodels 1 and 2 by the application of the assumptions and arguments of Mjelde (1975).

The advantages of the model, as compared with Markov-Lanchester methods, are commented on by Mjelde (1974). The time-dependent distributions of the losses cannot usually be obtained by the latter methods except by the numerical solution of a possibly large set of differential-difference equations describing state transitions; this may result in a compounding of errors and a long computer time. However, non-timedependent results, such as the winning probabilities and the distributions of survivors 
in a prolonged campaign, can be obtained, see Brown (1963), Connolly and Springall (1967), Springall (1968), and Smith (1965).

Following a description of an antisubmarine warfare scenario, three models are presented for respectively: the total losses, the tactical options of an individual submarine captain, and the losses inflicted upon the ships by an individual submarine. A concluding section comments on various applicational aspects, including the selection of convoy sizes.

\section{An antisubmarine warfare scenario}

In the scenario considered by Mjelde $(1974,1975)$, merchant ship convoys are crossing an ocean region. They are attacked by enemy submarines that operate independently of each other within an area across the convoy lanes. Each convoy is defended by surface and air screens that have some probability of destroying an approaching submarine. The submarines may be of different types (conventional and/or nuclear) and may carry weapons of different types (missiles and/or torpedoes). The number of ships destroyed, given a weapon release, is a random variable. The submarine attempts to escape after an attack, with some probability of being destroyed.

Koopman (1946) defined the region of approach of a submarine in relation to a convoy, as the set of positions from which the submarine is able to approach to a zero distance from the convoy, in dependency of the relative speeds of the submarine and the convoy. An encounter is said to occur if a submarine detects a convoy within the region of approach, and the captain decides to attack the convoy. The operation of a submarine is limited by various factors, such as the maximum time it can stay in the operating area and the total number of weapons on board; a submarine leaves the operating area before the maximum time has expired if the number of weapons remaining is insufficient for an attack.

It is assumed that only a small number of ships are destroyed, which means that a convoy maintains its identity throughout the battle.

\section{The total losses}

A general functional model for the composition of the losses of ships due to several individual submarines was given by Mjelde (1975) in terms of the following eqs. (1), (2) and (3).

Since the submarines are assumed to operate independently of each other, the total number of ships lost can be expressed by the convolution of the losses inflicted upon the ships by each submarine class (nuclear and/or conventional). For this reason only one class of submarines is considered in this paper.

The performance of a single submarine arriving at time $\theta$ is described by:

$V_{n}(\theta, t)$ : The probability that the submarine destroys $n$ ships in the time interval $[\theta, t], n=0,1,2, \ldots$

The probability generating function, the expected value, and the variance of the probability distribution $\left\{V_{n}(\theta, t)\right\}$ are denoted by $G(\theta, t, z), E(\theta, t)$ and $\sigma^{2}(\theta, t)$, respectively; then:

$$
G(\theta, t, z)=\sum_{n=0}^{\infty} V_{n}(\theta, t) z^{n}
$$


and $E(\theta, t)$ and $\sigma^{2}(\theta, t)$ can be obtained from the first and second derivatives of $G(\theta, t, z)$ with respect to $z$, evaluated at $z=1$.

The losses of ships are defined by their probability generating functions, expected values, and variances:

$$
\begin{aligned}
& M(t, z), x(t), \sigma_{x}{ }^{2}(t): \text { The total number of ships destroyed before time } t \text { by all } \\
& \text { submarines. }
\end{aligned}
$$

If the submarines arrive at the operating area according to a Poisson stream with rate $\mu(\theta)$ at time $\theta$, where $\mu(\theta)=0$ for $\theta \leqslant 0$, it follows from the arguments of Mjelde (1975) that:

$$
\begin{gathered}
M(t, z)=\exp \left\{\int_{0}^{t} \mu(\theta)[G(\theta, t, z)-1] d \theta\right\} \\
x(t)=\int_{0}^{t} \mu(\theta) E(\theta, t) d \theta \\
\sigma_{x}^{2}(t)=\int_{0}^{t} \mu(\theta)\left[\sigma^{2}(\theta, t)+E^{2}(\theta, t)\right] d \theta
\end{gathered}
$$

Fast Fourier-Laplace transform inversion procedures, see for instance Prescott and Jenkins (1974) or Uhrich (1969), can be applied in the determination of the probability distribution of the losses of ships at any given time, from the probability generating functions, as described in detail in Mjelde (1977).

Factors such as delays in the home port, speed variations, and navigation errors tend to randomize the arrivals of submarines at the operating area. If the successive arrival epochs of each individual submarine are considered as a renewal process, it can be shown that Poisson arrivals can emerge by the superposition of a large number of renewal processes; it is assumed that the inter-arrival times of each individual process (submarine), although arbitrarily distributed, have a large expectation, see Feller (1966).

\section{Tactical model}

Mjelde (1975) applied the general model (1), (2) and (3) to the determination of the losses of ships in a specific scenario where each submarine carried weapons of a single type. When a more complex battle scenario is analysed, the corresponding mathematical expression for the function $G(\theta, t, z)$ in eqn. (1) can easily become lengthy and unstructured; a simplification results if $G(\theta, t, z)$ is expressed in the form of the model defined by eqn. (11) of the following section. The purpose of the present section is to motivate the definitions of the quantities $N, \Omega, F(N, z), U(N, z)$ and $b(N, \theta, v)$ used in eqn. (11). This is done by the consideration of a specific battle scenario, for which the previously given quantities are expressed in terms of more detailed parameters, bearing in mind that analogous definitions, as well as the tactics model (11), are equally applicable to many other specific scenarios.

Consider a situation where submarines fire missiles and torpedoes repsectively from positions outside and inside the defending screens around a convoy. When a convoy is encountered a submarine equipped with missiles and torpedoes fires missiles. With respect to the use of torpedoes two tactical options are discussed:

(1) the submarine tries to penetrate the convoy screens to make a torpedo attack when missiles have been released and 
(2) the submarine leaves the convoy and does not fire torpedoes until all missiles have been expended.

A submarine with only torpedoes on board attempts to penetrate the screens of an encountered convoy.

Experience from the Second World War, see Falconer (1976), shows that there are three main factors affecting convoy losses: The convoy must be found, the screen of escorts must be penetrated, and the weapons must be fired. Large and small convoys are almost equally detectable, but the approach probability, Koopman (1946), is larger for large convoys due to their reduced speed. The probability of screen penetration depends on the linear escort density, which is higher for large convoys. Once inside the screen of a convoy, the submarine is very difficult to attack, and can fire all torpedoes in the tubes almost at leisure: any convoy offers more than enough targets. Reloading takes time and a submarine could rarely use more than a single load of weapons against a convoy.

The following detailed parameters are formulated according to the above observations:

I: The number of different types of weapons a submarine can use; $I=2$, missiles and torpedoes.

$\lambda$ : The rate of a Poisson process describing encounters between a single submarine and convoys.

$F_{i}(z)$ : The probability generating function of the number of ships destroyed by a completed release of a load of weapons of type $i$ against a convoy by a submarine.

$E_{i}, \sigma_{i}{ }^{2}$ : The expected value and variance of $F_{i}(z)$.

$T$ : The endurance-limited time a submarine can stay in the operating area searching for convoys.

$P_{1}$ : The probability that a submarine is not destroyed by the defences of an encountered convoy and reaches an attack position from which torpedoes are released.

$P_{2}$ : The probability that a submarine successfully escapes through the defences of an encountered convoy after weapons have been released.

$c$ : The probability that a submarine captain decides to fire torpedoes against a convoy, given that missiles have been fired from a position outside the screens and that torpedoes are available. $c=0$ or 1 according to the previously given tactical options.

$L_{i}$ : The maximum number of times a single submarine can release weapons of type $i$.

The number of ships destroyed in a convoy attack is assumed to be independent of the number of previous attacks against the convoy. This is in accordance with the observations described above. The number $L_{i}$ is equal to the total number of weapons of type $i$ on board a submarine divided by the number of weapons that is used in each release.

It will be assumed that $L_{1} \leqslant L_{2}$; analogous arguments apply if $L_{1}>L_{2}$. 
The number of weapons on board a submarine is determined by the following quantities:

$n_{i}$ : The number of times a submarine has released a load of missiles or torpedoes, $i=1,2$, against a convoy.

Define the vector:

$$
N=\left(n_{1}, n_{2}\right)
$$

and the intermediate quantities:

$b(N, \theta, t)$ : The conditional probability that a submarine is on patrol searching for convoys at time $t$, given the vector $N$ and arrival at the operating area at time $\theta$.

The probabilities $b(N, \theta, t)$ are first expressed in terms of the detailed parameters, and these probabilities are then used to determine the input $V_{n}(\theta, t)$ to the general model.

Since $L_{1} \leqslant L_{2}$ and a release of torpedoes is preceded by a release of missiles (if missiles are available), it is seen that $b(N, \theta, t)>0$ only if $N$ belongs to one of the following two sets:

$$
\begin{aligned}
& \Omega_{1}=\left\{N: n_{1}<L_{1}\right\} \\
& \Omega_{2}=\left\{N: n_{1}=L_{1} \text { and } n_{2}<L_{2}\right\}
\end{aligned}
$$

Then for $N \in \Omega_{1}$ :

$$
n_{2}= \begin{cases}0 & \text { if } c=0 \\ n_{1} & \text { if } c=1\end{cases}
$$

and for $N \in \Omega_{2}$ :

$$
n_{2}=\left\{\begin{array}{cc}
0, \ldots,\left(L_{2}-1\right) & \text { if } c=0 \\
L_{1}, \ldots,\left(L_{2}-1\right) & \text { if } c=1
\end{array}\right.
$$

It is useful to define:

$$
d(n, \lambda, \theta, t)= \begin{cases}\frac{(\lambda(t-\theta))^{n}}{n !} \exp (-\lambda(t-\theta)) & \text { for } t-\theta \leqslant T \\ 0 & \text { for } t-\theta>T\end{cases}
$$

Clearly $b(N, \theta, t)=0$ for $(t-\theta) \geqslant T$. If $(t-\theta)<T$ it follows that for $N \in \Omega_{1}$ :

$$
b(N, \theta, t)= \begin{cases}d\left(n_{1}, \lambda, \theta, t\right) & \text { for } c=0 \\ \left(P_{1} P_{2}\right)^{n_{1}} d\left(n_{1}, \lambda, \theta, t\right) & \text { for } c=1\end{cases}
$$

and for $N \in \Omega_{2}$ :

$$
b(N, \theta, t)= \begin{cases}\left(P_{1} P_{2}\right)^{n_{2}} d\left(n_{2}+L_{1}, \lambda, \theta, t\right) & \text { for } c=0 \\ \left(P_{1} P_{2}\right)^{n_{2}} d\left(n_{2}, \lambda, \theta, t\right) & \text { for } c=1\end{cases}
$$


In expressing $G(\theta, t), E(\theta, t)$ and $\sigma^{2}(\theta, t)$ in terms of the previously defined parameters it is convenient to introduce the following probability generating function:

$$
F(N, z)=\sum_{i=1}^{l} \alpha_{i}(N) F_{i}(z)=\sum_{j=0}^{\infty} f_{j}(N) z^{j}
$$

where

$$
\alpha_{1}(N)= \begin{cases}1 & \text { for } N \in \Omega_{1} \\ 0 & \text { for } N \in \Omega_{2}\end{cases}
$$

and

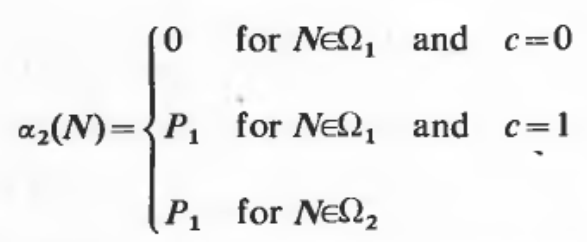

Thus $\alpha_{1}(N)$ is the probability that a submarine releases a load of weapons of type $i$, given an encounter with a convoy and given the matrix $N$, and $F(N, z)$ is the corresponding generating function of the losses of ships, with $f_{j}(N)$ being the probability that $j$ ships are destroyed. then:

Let $E(N)$ and $\sigma^{2}(N)$ be the expected value and variance derived from $F(N, z)$, and

$$
E(N)=\sum_{i=1}^{I} \alpha_{i}(N) E_{i}
$$

and

$$
\sigma^{2}(N)+E^{2}(N)=\sum_{i=1}^{I} \alpha_{i}(N)\left[\sigma_{i}{ }^{2}+E_{i}{ }^{2}\right]
$$

Define:

$$
\Omega=\Omega_{1} \cup \Omega_{2}
$$

and $U(N, z)$ and $g_{j}(N)$ by:

$$
U(N, z)=\prod_{i=1}^{\prime} F_{i}(z)^{n_{i}}=\sum_{i=0}^{\infty} g_{j}(N) z^{j}
$$

\section{The losses inflicted by a single submarine}

The previously given considerations of the operations of an individual submarine motivate the definition of a new model for the composition of the losses of ships due to several attacks by a single submarine. The resulting eqns. (11), (12) and (13) can be regarded as an intermediate model between the general model (1) and the specific tactical model of an individual submarine given in the previous section.

From the definition of the probabilities $V_{n}(\theta, t)$, it follows that:

$$
\begin{aligned}
V_{n}(\theta, t+\Delta t)= & {\left[V_{n}(\theta, t)-\sum_{N \in \Omega} b(N, \theta, t) g_{n}(N)\right] } \\
& +\sum_{N \in \Omega} b(N, \theta, t) g_{n}(N)\left[(1-\lambda \Delta t)+\lambda \Delta t f_{0}(N)\right] \\
& +\sum_{N \in \Omega} b(N, \theta, t) \sum_{j=0}^{n-1} g_{j}(N) \lambda \Delta t f_{(n-j)}(N)
\end{aligned}
$$


The equation can be justified if it is noted that a submarine arriving at the operating area at time $\theta$ can destroy $n$ ships before time $(t+\Delta t)$ in three mutually-exclusive ways:

(1) The submarine destroys $n$ ships before time $t$ and is not on patrol searching for convoys at time $t$.

(2) The submarine is on patrol at time $t$, having destroyed $n$ ships; there is either no encounter with the convoy in the succeeding time interval $\Delta t$ or there is an encounter and no ships destroyed.

(3) The submarine has destroyed $j$ ships before time $t$, where $0 \leqslant j \leqslant n-1$, and is searching for convoys at time $t$, and in the following time interval $\Delta t$ there is an encounter with a convoy and $(n-j)$ ships are destroyed in the corresponding attacks.

If the latter equation is rearranged, divided by $\Delta t$ and $\Delta t \rightarrow 0$, it becomes:

$$
\begin{aligned}
\frac{\partial V_{n}(\theta, t)}{\partial t}= & -\lambda \sum_{N \in \Omega} b(N, \theta, t) g_{n}(N) \\
& +\sum_{N \in \Omega} b(N, \theta, t) \sum_{j=0}^{n} g_{j}(N) \lambda f_{(n-j)}(N)
\end{aligned}
$$

Multiplication by $z^{n}$ and summing for all integers $n \geqslant 0$ gives $(\partial / \partial t) G(\theta, t, z)$ when the eqns. (7) and (10) are expressed by:

$$
\sum_{n=0}^{\infty} \sum_{j=0}^{n} g_{j}(N) f_{(n-j)}(N) z^{n}=U(N, z) F(N, z)
$$

Integration with the initial condition $G(\theta, \theta, z)=1$ yields:

$$
G(\theta, t, z)=1+\lambda \sum_{N \in \Omega} U(N, z)[F(N, z)-1] \int_{\theta}^{t} b(N, \theta, v) d v
$$

From the first and second derivatives of $G(\theta, t, z)$ with respect to $z$, evaluated at $z=1$, it follows that:

and

$$
E(\theta, t)=\lambda \sum_{N \in \Omega} E(N) \int_{\theta}^{t} b(N, \theta, v) d v
$$

$$
\sigma^{2}(\theta, t)+E^{2}(\theta, t)=\lambda \sum_{N \in \Omega}\left[\sigma^{2}(N)+E^{2}(N)+2 E(N) \sum_{i=1}^{l} n_{i} E_{i}\right] \int_{\theta}^{t} b(N, \theta, v) d v
$$

The quantity inside the brackets of eqn. (13) is the increase in the square of the number of ships that are destroyed due to an attack, given an encounter and given the vector $N$.

\section{Concluding remarks}

Explicit expressions for the losses defined by $M(t, z)$ can be determined if $\mu(\theta)=\mu=$ constant for all $\theta \geqslant 0$; however, although straight-forward to derive, the expressions are somewhat lengthy and are therefore not recorded here.

With a given total number of available escorts the number of escorts assigned to a convoy increases proportionally with convoy size. It follows that the linear escort density of a convoy increases with the square-root of the number of ships in the convoy. The encounter rate $\lambda$ is given by the product of four factors: the arrival rate of convoys, the probability that an arriving convoy is detected, the probability that the 
submarine is able to approach the convoy, and, finally, the probability that the captain decides to attack the convoy. According to Falconer (1976) only two of these factors may depend significantly on convoy size: the arrival rate is approximately inversely proportional to convoy size, and the approach probability may increase with convoy size, due to reduced convoy speed.

With the incorporation of the previously given considerations the compound model given by the equations (1) and (11) can be used to discuss the relative effectiveness of convoys of various sizes, and an 'optimal' convoy size can be determined according to specified decision criteria, such as the maximization of the probability that at least a given number of ships arrive safely at their destinations during a given planning period, or the minimization of the probability of the arrival of a small number of ships, insufficient for the support of the civilian or military logistical operations.

It has been assumed that the time duration of attacks is zero, or negligible in comparison with the time needed to search for convoys. Although this is frequently a realistic assumption, it could be removed by the introduction of the probability distribution of the duration of attacks, by the use of integral equations related to those of Mjelde (1974).

Finally, the attention of the reader is called to the applicability of the models of this paper to problem areas, such as: military scenarios where submarines are replaced by aircraft or other weapons; or the analysis of the effect of pollution, if submarines are replaced by sources of pollution.

\section{ACKNOWLEDGMENTS}

The work of this paper was supported by: The Norwegian Defence Research Establishment, Kjeller, Norway; SHAPE Technical Centre, The Hague, The Netherlands; and, SACLANT ASW Research Centre, La Spezia, Italy.

\section{REFERENCES}

BRown, R. H. (1963). Theory of combat: the probability of winning. Operations Research, 11, $418-425$

Connolly, B., and Springall, A. (1967). The overall outcome of a certain Lanchester combat model. Interim Technical Report 67/1, Dept. of Statistics and Statistical Laboratory, Research Division, Virginia Polytechnic Institute.

FalCoNER, N. (1976). On the size of convoys: an example of the methodology of leading wartime OR scientists. Operational Research Quarterly, 27, 315-327.

Feluer, W. (1966). An introduction to Probability Theory and its Applications (New York: Wiley).

Koopman, B. O. (1946). Search and screening. Operations Evaluation Group, Office of the Chief of Naval Operations, Washington D.C., OEG Report No. 56 ATI 64627.

MJELDE, K. M. (1974). An analytical approach to a class of battles. Operations Research, 22, 93-99.

MJELDE, K. M. (1975). On a supplementary time variable approach to battle analysis. Naval Research and Logistics Quarterly, 22, 189-196.

MJELDE, K. M. (1977). An analytical minefield evaluation model without space averages. Naval Research and Logistics Quarterly, 24, 639-650.

Prescott, J., and JenKINS, R. L. (1974). An improved fast Fourier transform. I.E.E.E. Transactions Acoustics, Speech and Signal Processing, 22, 226-227.

SmITH, D. G. (1965). The probability distribution of the number of survivors in a two-sided combat situation. Operational Research Quarterly, 16, 429-437.

Springall, A. (1968). Contributions to Lanchester combat theory. Ph.D. Thesis, Virginia Polytechnic Institute, Blackburg, Virginia.

Uhrich, M. L. (1969). Fast Fourier transforms without sorting, I.E.E.E. Transactions on Audio and Electroacoustics, AU-17, 170-172. 